\title{
Data-Driven Correlation Analysis Between Observed 3D Fatigue-Crack Path and Computed Fields from High-Fidelity, Crystal-Plasticity, Finite-Element Simulations
}

\author{
KYLE D. PIERSON ${ }^{1}$ JACOB D. HOCHHALTER, ${ }^{2}$ \\ and ASHLEY D. SPEAR (D) \\ 1.-School of Computing, University of Utah, Salt Lake City, UT, USA. 2.-NASA Langley Research \\ Center, Hampton, VA, USA. 3.-Department of Mechanical Engineering, 2151 Kennecott Building, \\ University of Utah, Salt Lake City, UT 84112, USA. 4.-e-mail: ashley.spear@utah.edu
}

\begin{abstract}
Systematic correlation analysis was performed between simulated micromechanical fields in an uncracked polycrystal and the known path of an eventual fatigue-crack surface based on experimental observation. Concurrent multiscale finite-element simulation of cyclic loading was performed using a highfidelity representation of grain structure obtained from near-field high-energy $\mathrm{x}$-ray diffraction microscopy measurements. An algorithm was developed to parameterize and systematically correlate the three-dimensional (3D) micromechanical fields from simulation with the 3D fatigue-failure surface from experiment. For comparison, correlation coefficients were also computed between the micromechanical fields and hypothetical, alternative surfaces. The correlation of the fields with hypothetical surfaces was found to be consistently weaker than that with the known crack surface, suggesting that the micromechanical fields of the cyclically loaded, uncracked microstructure might provide some degree of predictiveness for microstructurally small fatigue-crack paths, although the extent of such predictiveness remains to be tested. In general, gradients of the field variables exhibit stronger correlations with crack path than the field variables themselves. Results from the datadriven approach implemented here can be leveraged in future model development for prediction of fatigue-failure surfaces (for example, to facilitate univariate feature selection required by convolution-based models).
\end{abstract}

\section{INTRODUCTION}

Microstructural features play a governing role in the initiation and early stages of fatigue-crack growth. Variation in these features leads directly to variation in the paths and growth rates of microstructurally small cracks and, consequently, to scatter among fatigue lifetimes of structural components. Modeling this variability is critical given that most of the service life of fatigue-critical components can be consumed by initiation and growth of microstructure-sensitive cracks. Yet, these early stages of fatigue-crack evolution are difficult to model because of their complex dependence on a broad range of microstructural features and the tendency to exceed propagation rates of long cracks with equivalent nominal stress intensity factors. ${ }^{1}$ The reader is directed to Ref. 2 for an encompassing review of metallographic aspects of microstructural heterogeneities and their role in fatigue cracking. Similarly, a review of micromechanical descriptions of the effect of microstructural heterogeneities is given in Ref. 3 .

Empirically based fatigue-life models were developed to link variability in fatigue life to microstructural features that were directly observable and quantifiable. Early examples of these approaches, which are overviewed in Refs. 4-6, based fatigue models on microstructural characteristics such as inclusion size, shape, and location. ${ }^{7-9}$ While empirical approaches have provided foundational knowledge regarding microstructural effects on fatigue performance, the resulting correlations and applicability of the developed models are valid only 
within the domain of the measured data and experimental parameters (e.g., boundary conditions, cyclic-load ratio, etc.). The formative works of Wei and Harlow ${ }^{10,11}$ clearly illustrate the need to use experimentation to discover and formulate hypotheses regarding the micromechanics at hand, not to fit empirical parameters.

Over the past two decades, there has been a shift toward computationally modeling microstructural features to investigate their impact on fatigue-crack initiation and early propagation. Such efforts typically use crystal-plasticity formulations to incorporate elastic and plastic anisotropy and either statistically representative or directly replicated microstructural domains to capture heterogeneities. For example, Bozek et al. ${ }^{12}$ simulated the effect of cyclic loading on cracking of second-phase particles. Subsequently, Hochhalter et al. ${ }^{13,14}$ used fatigue indicator parameters to predict which cracks would extend beyond those cracked particles. Twin boundary crack initiation sites, and their dependence on local microstructure, were presented by Yeratapally et al. ${ }^{15}$ Fatigue indicator parameters were used by Musinski et al. ${ }^{16}$ and Castelluccio et al.$^{17}$ to develop models for the subsequent propagation of cracks across a polycrystal. An encompassing study of the fatigue indicator parameters proposed to date is provided by Rovinelli et al. ${ }^{18}$ These studies represent a small sampling of high-fidelity, microstructure-sensitive fatigue modeling; a more complete review can be found in Refs. 19-22.

Advancements in these micromechanical modeling methods are being closely coupled with volumetric interrogation methods. This coupled approach provides a capability whereby parameters that cannot be measured directly can be computed in a simulation that serves to replicate the particular microstructure of the specimen. Spear et al. ${ }^{23}$ used near-field high-energy x-ray diffraction (HEDM) to measure grain morphology in a sample of Al 6061-T6. These data were then used to generate a finite-element model, which replicated the as-measured grain structure and crack morphology. Rovinelli et al. ${ }^{18}$ used diffraction contrast and phase contrast tomography to measure microstructure morphology and evolved crack faces in a near- $\beta \mathrm{Ti}$ alloy. Those data were used to generate a 3D fast Fourier transform (FFT) simulation with crystal plasticity. Bayesian networks were then used to analyze the correlation between the proposed short-crack driving forces and experimental observations.

As highlighted by the aforementioned studies, integrating x-ray-based measurement methods with high-fidelity simulation tools is providing a promising new approach to developing models for shortcrack propagation. ${ }^{24} \mathrm{New}$ focus is on efficient processing of these data (which can be quite large and complex) to extract useful information using datadriven approaches. In light of this, the objective of this paper is to describe a systematic, data-driven, correlation analysis between computed micromechanical fields of an uncracked, cyclically loaded 3D polycrystal and the known path of a $3 \mathrm{D}$ fatiguefailure surface based on direct observation from prior experimental measurements.

\section{MATERIALS AND METHODS}

\section{Experimental Measurements and Mesh Generation from Prior Work}

The data used in this work are derived from experimental measurements ${ }^{25}$ of $3 \mathrm{D}$ fatigue crack propagation within a grain-mapped volume of an $\mathrm{Al}-$ Mg-Si alloy. In that work, a naturally nucleated fatigue crack was propagated to failure under cyclic loading. The material volume above and below the failure surface was characterized post mortem using x-ray computed tomography and near-field HEDM. The former provided a high-resolution reconstruction of the failure surface, while the latter provided 3D grain maps adjacent to the failure surfaces, which can be seen in Fig. 1. Of the entire measured crack surface, $31.8 \%$ was found to be approximately normal (within $20^{\circ}$ ) to the global loading direction. Additionally, $41 \%$ and 59\% of the entire crack surface was deemed to be intergranular and transgranular, respectively, with the transgranular crack surface occurring along a wide variety of crystallographic planes.

The two halves of post mortem data were then virtually merged to generate a conformal finiteelement mesh that represents explicitly and with high fidelity the internal grain boundaries and incremental crack surfaces measured from experiment, as detailed in Ref. 23. In this work, the conformal finite-element mesh representing the uncracked microstructure was used to obtain computed micromechanical fields, which were then correlated with the reconstructed failure surface. Figure 1 summarizes the prior relevant work.

\section{Numerical Simulation of Cyclic Loading Applied to Uncracked Specimen}

A concurrent multiscale finite-element model was used to simulate cyclically applied displacement on the fatigue specimen tested in previous work. ${ }^{23}$ The previously generated mesh from Ref. 23 consists of a local, polycrystalline region representing the uncracked microstructure and a global region representing the geometry of the fatigue specimen, shown in Fig. 2. A mesh convergence study, detailed in Ref. 26, was carried out to ensure that both the global force-displacement response and local stresses, strains, and accumulated slip along an arbitrary query path through the polycrystalline domain converged sufficiently. The converged, multiscale mesh comprised $11.86 \mathrm{M}$ quadratic tetrahedral elements. 


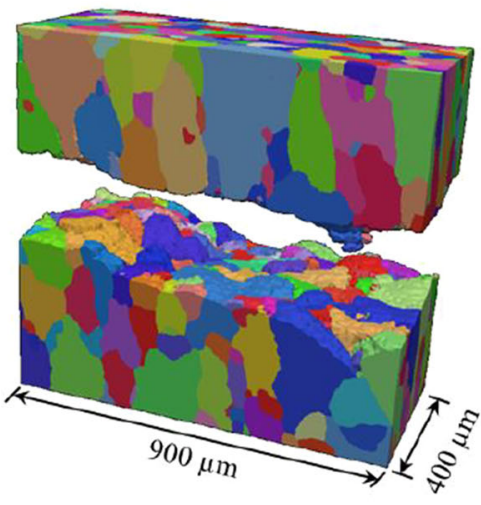

(a)

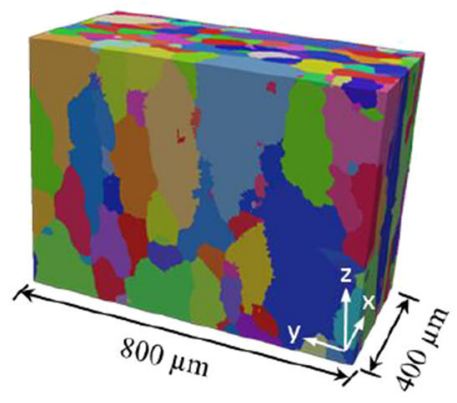

(b)
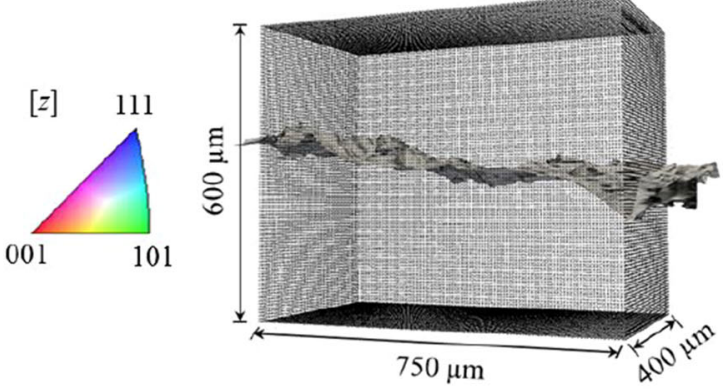

(c)

Fig. 1. (a) Post mortem reconstructions from an Al-Mg-Si alloy fatigue specimen based on near-field HEDM. (b) Approximation of uncracked volume and (c) reconstructed fatigue-failure surface from x-ray CT. The reference coordinate system is shown on the uncracked microstructural volume. Adapted with permission from Refs. 23,25.
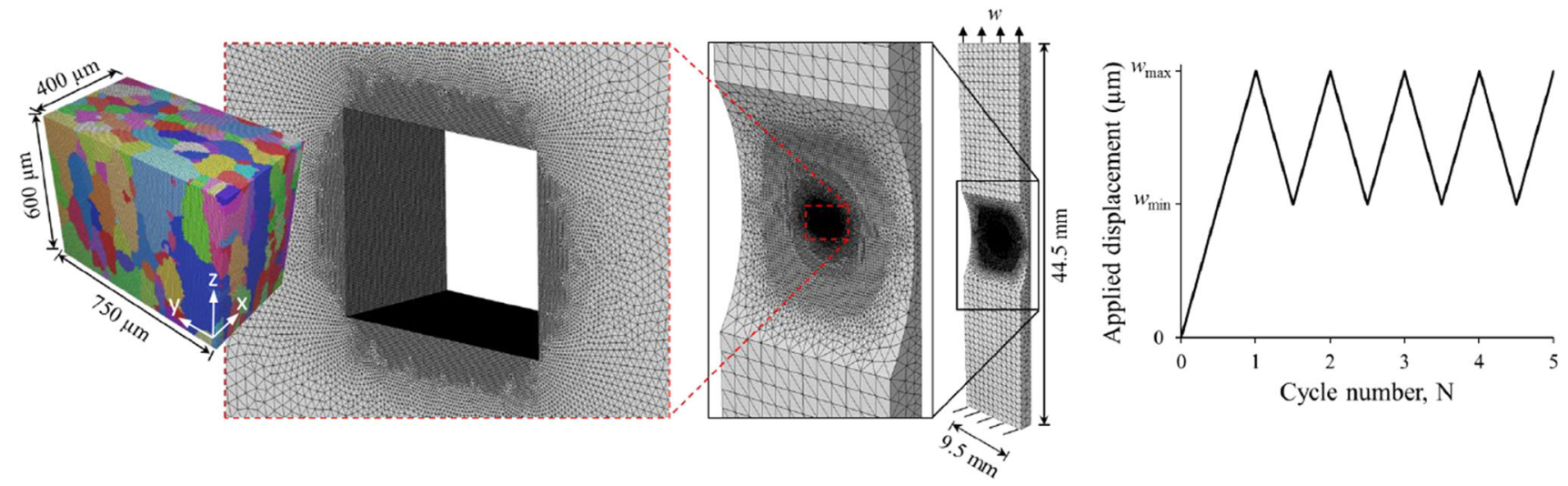

Fig. 2. Concurrent multiscale finite-element mesh and applied boundary conditions. The reference coordinate system is shown on the uncracked microstructural volume. Adapted with permission from Ref. 23.

A crystal, elasto-viscoplastic, constitutive model based on the implementation by Matous and Maniatty $^{27}$ was applied to the polycrystalline domain, and a J2-plasticity model was applied to the global domain. Both models were calibrated to ensure that the nominal (averaged) stress-strain behavior matches experimental data for the same material and that the simulated macroscopic strain fields in the notch region match those from digital image correlation measurements, which are described elsewhere. $^{26}$ The crystal, elasto-viscoplastic, constitutive model is capable of predicting inhomogeneous deformation and stress fields that arise at mesoscale as a result of interactions among discrete grains. In the model, plastic deformation is manifested by slip evolution on 12 octahedral slip systems $(\{111\}\langle 110\rangle)$. All elements within the polycrystalline domain are assigned the same material properties; however, each element is assigned a crystal orientation based on the grain to which that element belongs. The crystal orientations are derived directly from the near-field HEDM measurements described above. The calibrated parameters for both constitutive models are provided in Table I. Properties for the J2-plasticity model include the elastic modulus $(E)$, Poisson's ratio $(v)$, yield strength $\left(\sigma_{y}\right)$, and hardening modulus $\left(h^{\prime}\right)$. Properties for the crystal-plasticity model include a reference slip rate $\left(\dot{\gamma}_{0}\right)$, a hardening rate-sensitivity parameter $(m)$, a hardening-rate parameter $\left(G_{0}\right)$, and initial hardness $\left(g_{0}\right)$.

Boundary conditions were applied to replicate constraints and loading applied in the actual experiment. Namely, the grip ends of the specimen are constrained from displacing in the $x$ and $y$ directions. The lower grip end is further constrained from displacing in the $z$ direction. The upper grip end is subjected to vertical displacement, $w$, which cycles between $w_{\max }=65 \mu \mathrm{m}$ and $w_{\min }=38 \mu \mathrm{m}$. The values for applied displacement were selected to reproduce the applied loading from experiment, detailed in Ref. 25. 
Table I. Calibrated material parameters

\begin{tabular}{|c|c|c|c|c|c|c|c|}
\hline \multicolumn{4}{|c|}{ J2-plasticity model } & \multicolumn{4}{|c|}{ Crystal plasticity model } \\
\hline$\underline{E}(\mathbf{M P a})$ & $v$ & $\underline{\sigma_{\boldsymbol{y}}(\mathbf{M P a})}$ & $\underline{\boldsymbol{h}^{\prime}(\mathbf{M P a})}$ & $\underline{\dot{\gamma}_{\mathbf{0}}\left(\mathbf{s}^{-\mathbf{1}}\right)}$ & $m$ & $\underline{G_{0}(\mathbf{M P a})}$ & $\underline{g_{0}(\mathbf{M P a})}$ \\
\hline 70,326 & 0.33 & 206.5 & 1200 & 0.05 & 0.0049 & 150.0 & 95.5 \\
\hline
\end{tabular}

Numerical simulations were performed using the parallelized finite-element code ScIFEN. ${ }^{28}$ In total, five load cycles were simulated. The following list summarizes all local variables that were recorded for the entire polycrystalline domain at the beginning and end of each simulated load cycle:

$D_{1} \quad$ Maximum value of accumulated slip among the 12 octahedral slip systems

$D_{2} \quad$ Maximum value of total accumulated slip over each slip plane

$D_{3} \quad$ Accumulated slip summed over all slip systems

$D_{4} \quad$ Maximum value of energy dissipated on a given slip plane during plastic deformation

$D_{5} \quad$ Modified Fatemi-Socie parameter

$\bar{\epsilon} \quad$ Symmetric strain tensor composed of $\epsilon_{x x}$, $\epsilon_{y y}, \epsilon_{z z}, \epsilon_{x y}, \epsilon_{x z}$, and $\epsilon_{y z}$

$\epsilon_{1} \quad$ Principal eigenvalue of the strain tensor

$\epsilon_{v m} \quad$ von Mises strain

$\bar{\sigma} \quad$ Symmetric stress tensor composed of $\sigma_{x x}$, $\sigma_{y y}, \sigma_{z z}, \sigma_{x y}, \sigma_{x z}$, and $\sigma_{y z}$

$\sigma_{1} \quad$ Principal eigenvalue of the stress tensor

$\sigma_{u m} \quad$ von Mises stress

$M^{\text {micro }} \quad$ Micromechanical Taylor factor ${ }^{29}$

The variables $D_{1, \ldots, 5}$ represent slip-based damage metrics described by Hochhalter et al. ${ }^{13}$ and implemented within the ScIFEN framework. Additionally, the micromechanical Taylor factor, $M^{\text {micro }}$, was computed throughout the polycrystalline domain based on the work of Raabe et al., ${ }^{29}$ as follows:

$$
M^{\text {micro }}=\frac{D_{3}}{\epsilon_{v m}}
$$

In Eq. $1, D_{3}$ is the summation over all $N_{s}$ slip systems of the slip accumulated on each slip system, $\alpha$, throughout cyclic loading:

$$
D_{3}=\sum_{\alpha=0}^{N_{s}} \int_{0}^{t}\left|\dot{\gamma}^{\alpha}\right| \mathrm{d} t,
$$

where $\dot{\gamma}^{\alpha}$ is the slip rate on a given slip system. The term $\epsilon_{v m}$ represents the local von Mises equivalent strain, which is computed as

$$
\epsilon_{v m}=\sqrt{\frac{2}{3} \bar{\epsilon}: \bar{\epsilon}}
$$

The variables in the above list, along with the cyclic changes in those variables, were included in the systematic correlation analysis.

\section{Convergence of Cyclic Field Variables}

The convergence of field variables was assessed by considering the change in each variable throughout the entire polycrystalline domain as a function of cycle count. For any given variable, $\lambda$, its cyclic value was computed at each point in the model based on the change in that variable from the minimum to maximum displacement over a given loading cycle. The change in each cyclic value was also computed between successive loading cycles. In other words, at each point in the model, and for all variables in a given cycle, $N$ :

$$
\begin{gathered}
\Delta \lambda_{N}=\lambda_{w_{\max }, N}-\lambda_{w_{\min }, N}, \\
\Delta^{2} \lambda_{N}=\Delta \lambda_{N}-\Delta \lambda_{N-1} .
\end{gathered}
$$

Figure 3 illustrates the convergence of $\Delta \sigma_{z z}$ and a visualization of $\Delta^{2} \sigma_{z z}$ approaching zero (similar convergence being verified for all variables). Convergence of the cyclic field variables implies that the results taken from the fifth loading cycle sufficiently represent the state of the polycrystalline domain to perform a meaningful correlation study.

\section{Correlation Analysis}

The finite-element results were first converted to a format amenable to performing the correlation analysis. Results associated with the fifth loading cycle were subsampled from the unstructured finiteelement mesh onto a $383 \times 750 \times 600 \mu \mathrm{m}^{3}$ grid with $1 \mu \mathrm{m}$ spacing between points. This was done for all field variables, producing a scalar-valued grid for each variable, $\lambda$, corresponding to peak load, and for each cyclic value, $\Delta \lambda$.

Additionally, the spatial gradients of every $\lambda$ and $\Delta \lambda$ variable were calculated and included in the correlation analysis. Here, the gradients of $\lambda$ and $\Delta \lambda$ at each point in the model were computed based on finite differences in the $y$ and $z$ directions (reminiscent of $2 \mathrm{D}$ image slices through the volume) using $h=3 \mu m$ spacing,* after which the $L_{2}$ norm was taken to produce a scalar value. In the following equations, subscripts indicate the grid coordinates of a given point.

\footnotetext{
*Values of $1 \mu \mathrm{m}$ and $3 \mu \mathrm{m}$ were considered for $h$, with the latter being equal to half the size of the discretization for the quadratic finite-element mesh. Ultimately, $h=3 \mu \mathrm{m}$ was found to provide stronger correlations.
} 

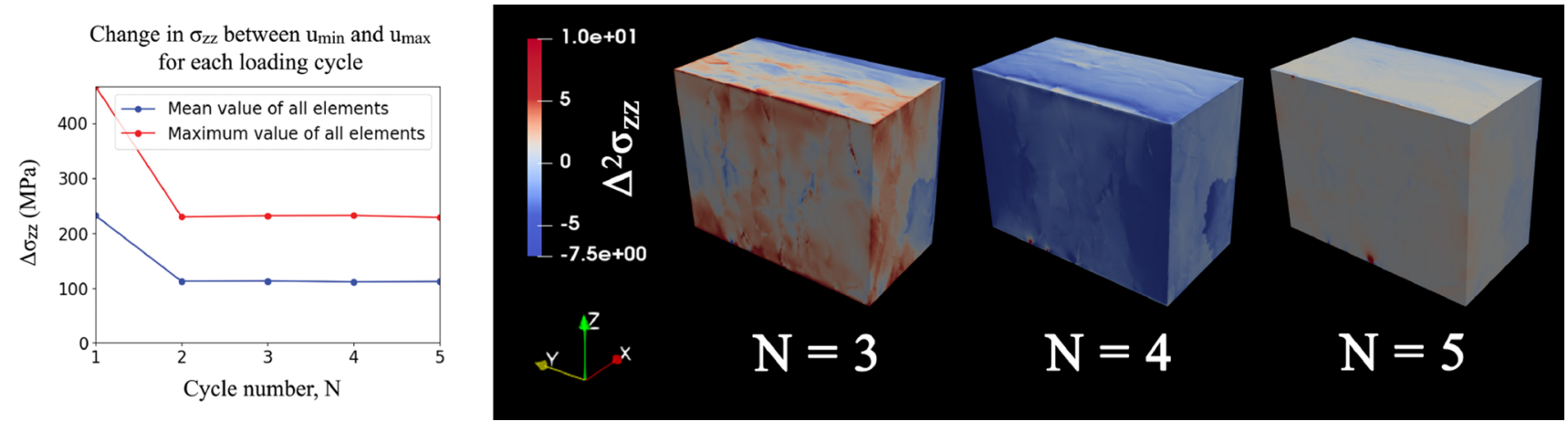

Fig. 3. Convergence of $\Delta \sigma_{z z}$ and $\Delta^{2} \sigma_{z z}(\mathrm{MPa})$ during cyclic loading.

$$
\frac{\mathrm{d} \lambda_{x, y, z}}{\mathrm{~d} y}=\frac{\lambda_{x, y+h, z}-\lambda_{x, y-h, z}}{2 h}, \quad \frac{\mathrm{d} \lambda_{x, y, z}}{\mathrm{~d} z}=\frac{\lambda_{x, y, z+h}-\lambda_{x, y, z-h}}{2 h}
$$

The next step is to represent the $3 \mathrm{D}$ crack surface, which was previously reconstructed from post mortem x-ray CT data and aligned with the uncracked microstructural domain, as a $2 \mathrm{D}$ grid of elevation values. This was accomplished by initializing a $383 \times 750 \mu \mathrm{m}^{2}$ grid with $1 \mu \mathrm{m}$ spacing between points, then assigning to each point an interpolated value of the corresponding $z$ coordinate of the crack surface, resulting in a height map. The interpolation method used was inverse bilinear interpolation. For a given point in the $x-y$ grid plane, the corresponding height-map value of the crack surface was used to identify neighboring points in the $3 \mathrm{D}$ scalar-valued grids.

In total, there were 88 scalar-valued grids to consider in the correlation analysis: one for each field variable, $\lambda$, listed in "Numerical Simulation of Cyclic Loading Applied to Uncracked Specimen" section; one for each cyclic variable, $\Delta \lambda$; and one for the gradient values of both $\lambda$ and $\Delta \lambda$. Each grid consisted of $383 \times 750 \times 600=1.724 \times 10^{9}$ data points derived from high-fidelity numerical simulation of the uncracked microstructure. There were an additional $383 \times 750=2.87 \times 10^{5}$ data points derived from the experimentally observed fatiguefailure surface.

Using this as input data, the goal of the algorithm implemented here is to determine-with minimal prior assumptions-which micromechanical field variables are correlated with the fatigue-crack path. The method chosen here is to compute the correlation between the value of a given variable at a particular point in the microstructure and its vertical distance to the crack surface. Only a local neighborhood around the crack surface is considered, i.e., a region into which the crack could plausibly have grown from any given configuration. The value of $L$ was systematically varied, and ultimately a value of $L=25 \mu \mathrm{m}$ (approximately $25 \%$ of the average grain diameter) above and below

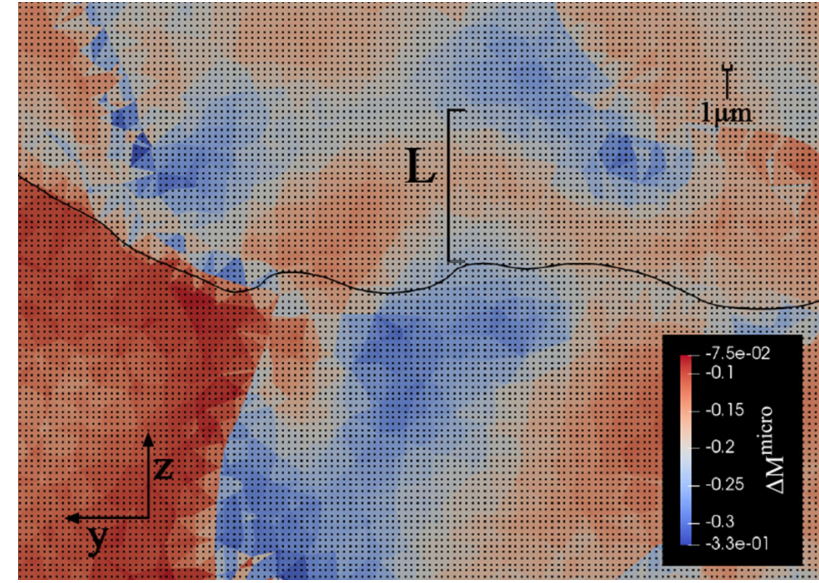

Fig. 4. Cyclic micromechanical Taylor factor computed for the uncracked polycrystal, shown at a particular slice through the volume. Superimposed is the trace of the actual crack surface from x-ray CT imaging. Also superimposed is the grid used for correlation analysis. The neighborhood of influence is defined by the distance $L$ above and below the crack surface.

the crack surface was selected to optimize the correlation results. This value was used in all correlation analyses described throughout this paper. The definition of $L$ is shown in Fig. 4 for a slice and subsection of the grid defined for $\Delta M^{\text {micro }}$. The grid points are shown in black with spacing of $1 \mu \mathrm{m}$, and the finite-element results obtained using an unstructured mesh are shown in the background. The trace of the actual crack surface is superimposed for reference.

Of this subset of points within $L$ of the surface, only regions where the gradient of a given field variable was sufficiently high were considered. This filter was implemented due to the propensity of some variables to exhibit near-zero change within the neighborhood, $L$. In such cases, the correlation of that variable with the distance to the crack surface does not add value to the analysis. In order for a gradient of a variable to be considered sufficiently high, it must be at least $t \%$ of its value at the same point. Here, $t$ was determined independently for each variable by finding the value of $t$ that 
maximized the correlation of that variable, while still retaining at least $10 \%$ of the total crack surface (see Fig. 5). For example, $t=8 \%$ for $\epsilon_{z z}$, while $t=11 \%$ for $\Delta D_{3}$. This implementation guarantees that regions contributing to the correlation analysis are not overly sparse, yet contain $\lambda$-values that are highly informative.

This method can be easily parallelized, as computations are performed on each point of the crack surface independently of all other points. Since computations must be performed for each of the $88 \times 383 \times 750 \times 50=1.264 \times 10^{9}$ points, such parallelization is a necessity. The data were loaded into shared memory accessible by all processes, after which the code was run on multiple cores. The entire algorithm is described in Fig. 6.

The results from the algorithm consist of data frames, where each row is an observed point from the microstructure and each column is the value of a field variable, or distance to the crack surface in the case of the last column. These data frames were then imported into $R^{30}$ which provides robust libraries for correlation analyses and visualizations. Pearson correlation coefficients were computed for each column with respect to the final column, then visualized for comparison.

\section{RESULTS AND DISCUSSION}

The computed fields for all 22 metrics and their respective cyclic values are visualized for the uncracked microstructure in Supplementary Figs. S1-S8 (online version). The computed correlation coefficients are shown in Fig. 7. A negative correlation indicates that, as the distance to the crack surface decreases, the variable of interest increases, and vice versa.

The correlation coefficients shown in Fig. 7 are not, at first look, overwhelmingly high. The challenge in assessing the statistical significance of these correlations is that the independent and identically distributed assumption required by a

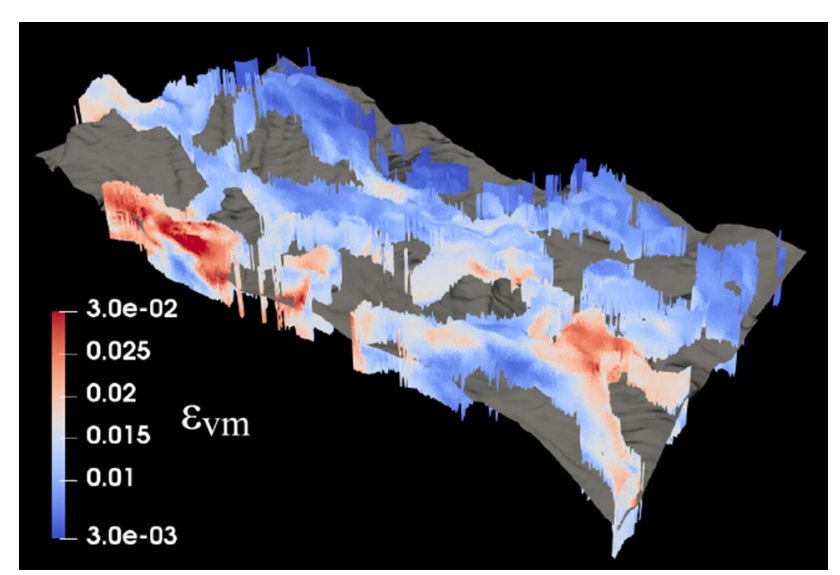

Fig. 5. Colored data points within $L$ vertical distance of the crack surface that meet the spatial-gradient threshold for inclusion in the correlation analysis of $\epsilon_{v m}$. typical $t$-test has been violated. A nonparametric measure, such as bootstrapping ${ }^{31}$ or using Spearman rank, could potentially help resolve this problem. An alternative approach to determine whether the correlation values in Fig. 7 are meaningful (but not necessarily statistically significant) is to apply the same correlation algorithm to alternative surfaces that could serve as hypothetical crack paths through the microstructure. Four alternative paths were considered: two $z$-normal planes positioned $200 \mu \mathrm{m}$ above and below the lower and upper faces, respectively, of the microstructural domain, and two instances of the measured crack surface offset by $125 \mu \mathrm{m}$ above and below the known crack path. Figure 8 shows the results for the latter two cases. Results for the two $z$-normal planes are provided in Supplementary Fig. S9 (online version). In all four cases, the correlations are consistently weaker than those for the actual crack surface, providing some evidence that micromechanical fields from a cyclically loaded, uncracked microstructure tend to correlate with the actual path of the $3 \mathrm{D}$ fatigue crack.

As shown in Fig. 7, the crack path is generally shown to be more strongly correlated with the spatial-gradient values than with the field variables at peak load or with the cyclic field variables, suggesting that the eventual fatigue crack sought paths of high gradients in stress and/or strain space. Considering only the gradient-based parameters, $D_{3}$ and $D_{5}$ exhibit the strongest correlation with the crack path among all the slip-based damage metrics, although the difference is relatively marginal. This indicates that the combined effect of slip activity on multiple slip systems $\left(D_{3}\right)$ as well as the combined effect of crystallographic slip and tensile stress on a slip plane $\left(D_{5}\right)$ play a more significant role in predicting the crack path than just the maximum value of slip on a single slip system or slip plane. While it seems reasonable for some of the metrics to have a relatively strong correlation with crack path (e.g., $\nabla\left(\epsilon_{z z}\right), \nabla\left(\Delta \epsilon_{v m}\right)$, and $\left.\nabla\left(\Delta \epsilon_{1}\right)\right)$, there are other correlations that are not immediately intuitive (e.g., $\nabla\left(\epsilon_{x x}\right)$ and $\nabla\left(\Delta \sigma_{y y}\right)$ ). Clearly, the factors affecting crack growth are highly complex, and one cannot rely on treating all field variables as independent mechanisms. As such, it is likely that there exists some complex combination of variables that serves to accommodate, promote, or hinder crack propagation, which corroborates previous conclusions in literature (e.g., Refs. 14, 18, 20). It will require further investigation using, for example, machine learning to understand how interaction of the variables leads to such apparent correlations.

Since the discontinuity of the crack is not modeled in this work (as was done in previous work ${ }^{23}$ ), the micromechanical fields computed here do not account for stress redistribution due to formation of new traction-free surface area; nor do they account for plastic zones or stress concentrations 


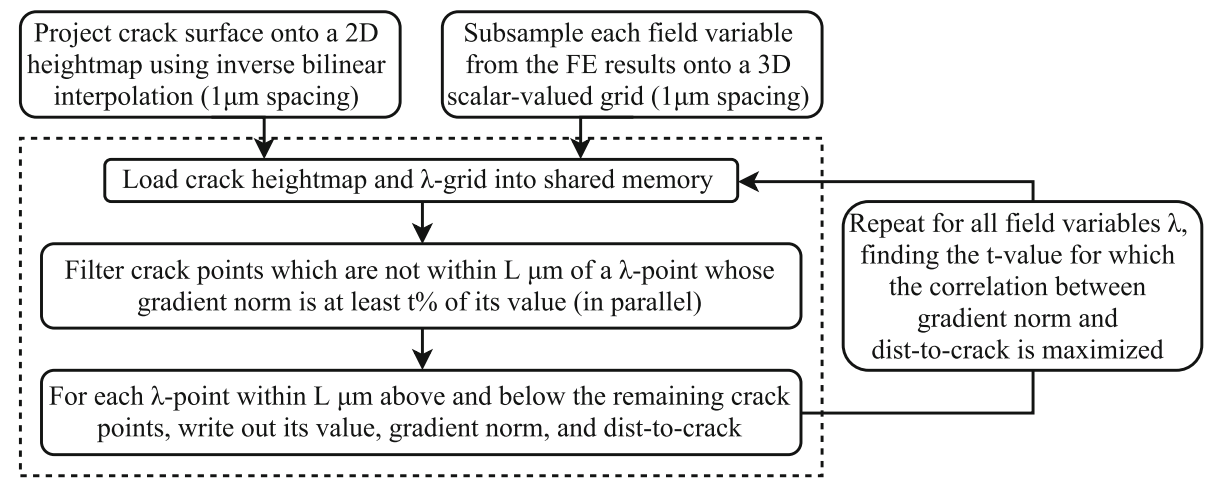

Fig. 6. Data extraction algorithm.

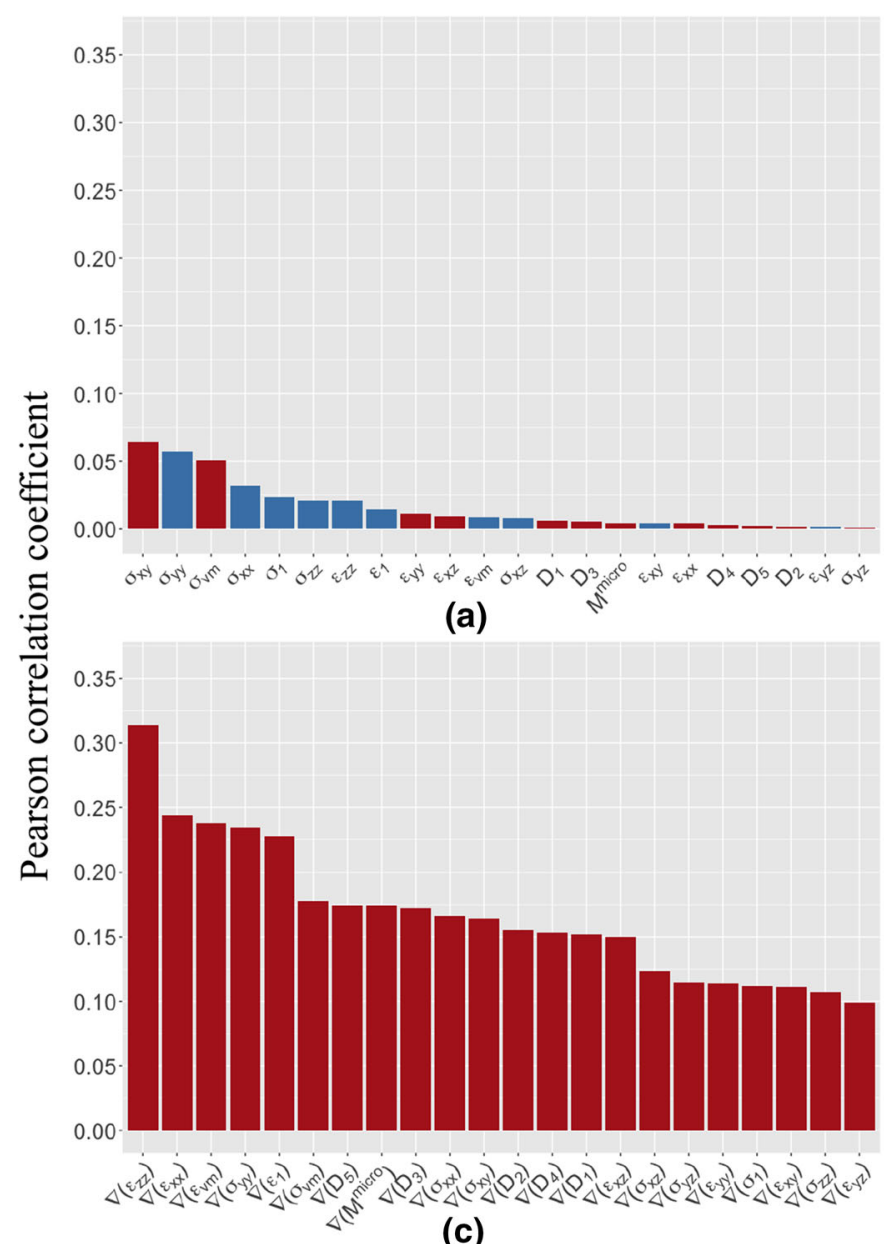

(c)

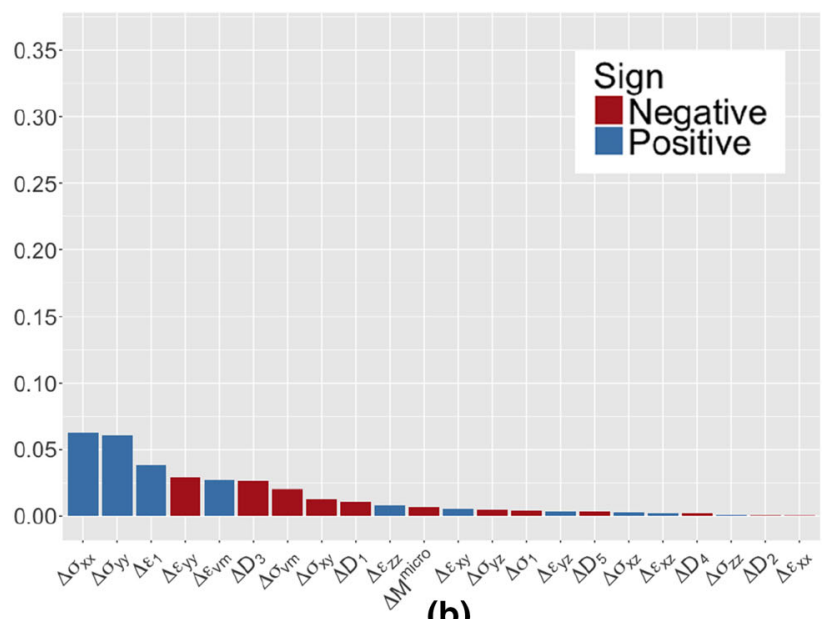

(b)

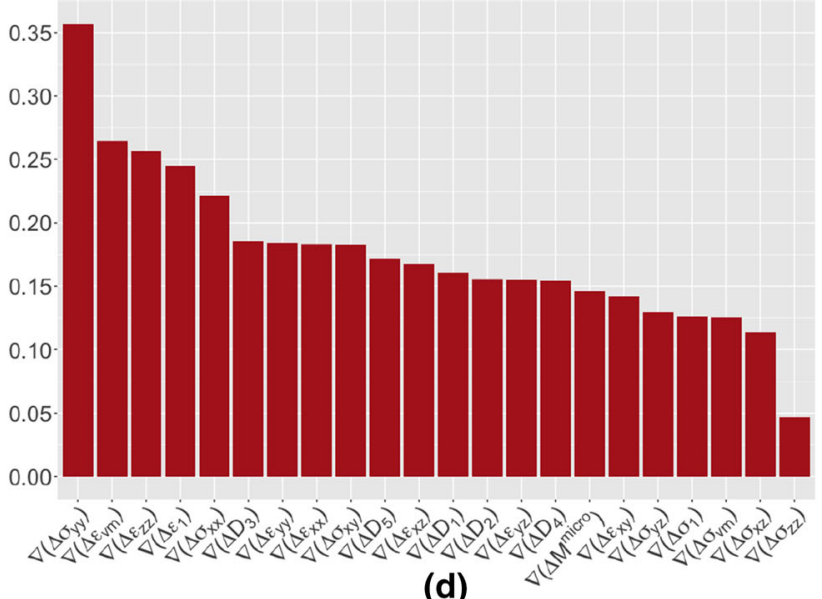

(d)

Fig. 7. Correlation coefficients computed between the following metrics and distance to crack surface: (a) field variables, $\lambda$; (b) cyclic change in field variables, $\Delta \lambda$; (c) spatial gradient of field variables, $\nabla(\lambda)$; and (d) spatial gradient of cyclic field variables, $\nabla(\Delta \lambda)$.

in the vicinity of a crack front. However, the objective is to identify what, if any, correlations exist between micromechanical fields in an uncracked microstructure and the 3D path of an eventual fatigue-crack surface. The implications of relatively strong correlations could be significant, in that the crack path might be approximated prior (or without having) to incorporate geometrically explicit crack representations. It is expected that such correlations would be relatively strong within a limited spatial domain surrounding the crack-nucleation site. Future work could investigate the size of this domain, beyond which the correlations are expected to diminish.

The analysis from this work could provide insight into the extraction of relevant features for predictive machine-learning models. In models where univariate feature selection is required, such as 


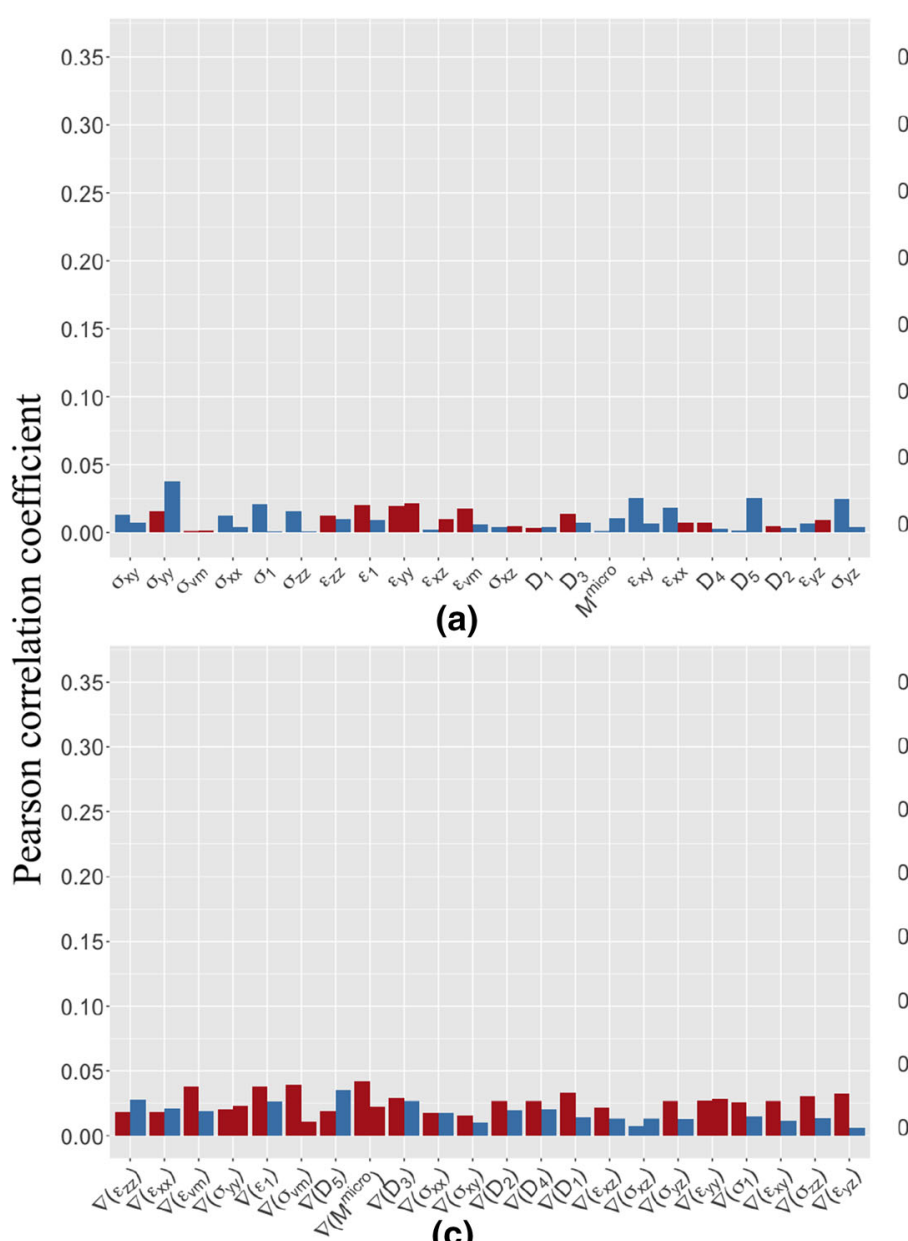

(c)

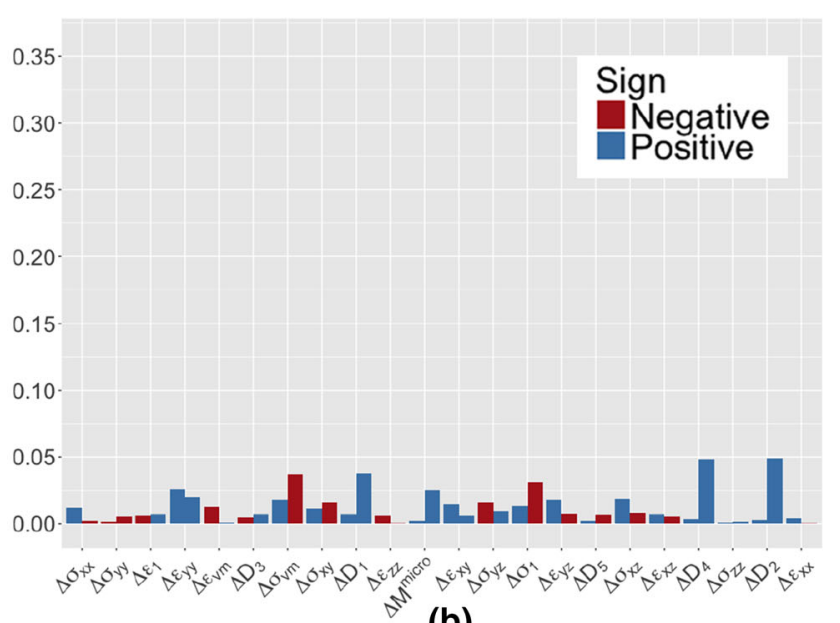

(b)

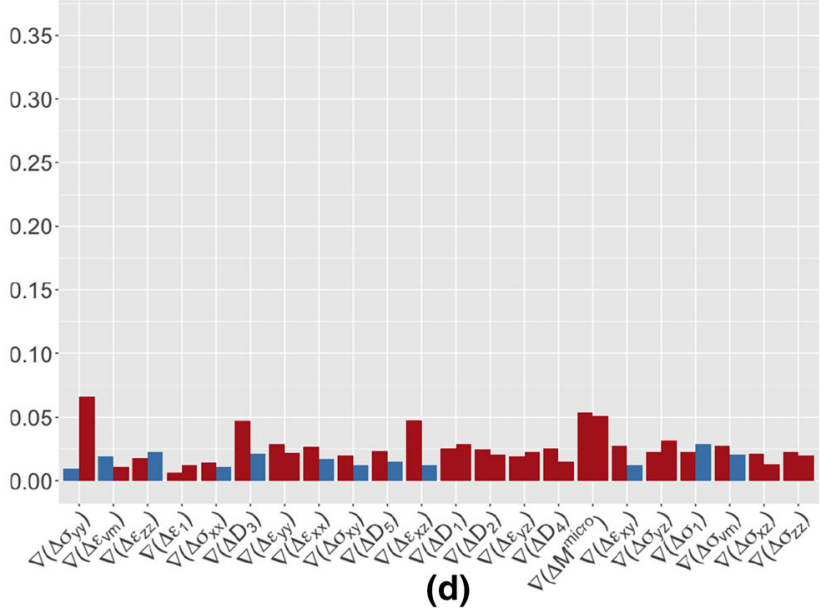

Fig. 8. Correlation coefficients computed between the following metrics and distance to hypothetical crack surfaces located $125 \mu \mathrm{m}$ below (left bars) and $125 \mu \mathrm{m}$ above (right bars) the known crack surface: (a) field variables, $\lambda$; (b) cyclic change in field variables, $\Delta \lambda$; (c) spatial gradient of field variables, $\nabla(\lambda)$; and (d) spatial gradient of cyclic field variables, $\nabla(\Delta \lambda)$.

convolution-based networks that infer useful information based on a grid of scalars, choosing the correct variables to use as a representation is critical. With new insight from the correlation analysis presented here, ongoing studies by the authors focus on the use of machine learning to identify critical combinations of, and relationships among, the most correlated variables with the evolution of fatigue-crack surfaces.

\section{CONCLUSION}

This work presents a systematic, data-driven approach to parameterize and correlate local micromechanical fields computed for an uncracked, cyclically loaded specimen with the known 3D fatigue-crack path observed from experiment. Specifically, local micromechanical field variables in the vicinity of an eventual crack surface are correlated with distance to that eventual surface. The intent is to identify whether the response of the uncracked microstructure subjected to realistic farfield loading might provide any predictive power in identifying the path of a fatigue-failure surface, which could have implications for future modeling efforts. In this work, a total of 88 micromechanical parameters, and $1.264 \times 10^{9}$ data points, are considered in the analysis. Thus, the data used here are considered to be large and rich in nature, albeit for just a single specimen. The parameters include field variables and slip-based damage metrics computed at peak load, as well as their corresponding cyclic values. Also considered are the gradients of all previously mentioned parameters. The micromechanical parameters, taken at discrete points, are then correlated with the distance from a given point to the known crack surface.

In general, the gradients of the micromechanical field variables appear to exhibit a stronger correlation with crack path than the field variables, themselves. This supports the claim that fatigue cracks generally seek paths of high gradients of stress, strain, or both. The variables, treated independently, are not sufficient to fully describe evolution of fatigue-crack surfaces. However, the systematic correlation analysis from this work 
provides insight into the extraction of relevant features for future development and testing of predictive models.

\section{ACKNOWLEDGEMENTS}

This material is based on research sponsored by the Air Force Office of Scientific Research Young Investigator Program, under Agreement No. FA9550-15-1-0172. The authors gratefully acknowledge Drs. Ravi Chona, Ben Smarslok, and Brian Gockel of the Air Force Research Laboratory for their support of the work. The support and resources from the Center for High Performance Computing at the University of Utah are gratefully acknowledged.

\section{ELECTRONIC SUPPLEMENTARY MATERIAL}

The online version of this article (https://doi.org/ 10.1007/s11837-018-2884-2) contains supplementary material, which is available to authorized users.

\section{REFERENCES}

1. R. Ritchie and J. Lankford, in Small Fatigue Cracks (The Metallurgical Society INC., Warrendale, 1986).

2. S.V. Narayana Murty, S.K. Manwatkar, and P.R. Narayanan, Mater. Perform. Charact. 6, 311 (2017).

3. H. Mughrabi, Philos. Trans. R. Soc. Lond. A: Math. Phys. Eng. Sci. 373, 2038 (2015).

4. Y. Murakami and M. Endo, Int. J. Fatigue 16, 163 (1994).

5. A. Fatemi and L. Yang, Int. J. Fatigue 20, 9 (1998).

6. K. Hussain, Eng. Fract. Mech. 58, 327 (1997).

7. P.J. Laz and B.M. Hillberry, Int. J. Fatigue 20, 263 (1998).

8. J.R. Brockenbrough, A.J. Hinkle, P.E. Magnusen, and R.J. Bucci, in Proceedings, FAA/NASA International Symposium on Advanced Structural Integrity Methods for Airframe Durability and Damage Tolerance (C. Harris, ed.), vol. NASA Conference Publication 3274, Part I, pp. 71-84, (Sep 1994).

9. M.A. Przystupa, R.J. Bucci, P.E. Magnusen, and A.J. Hinkle, Int. J. Fatigue 19, 285 (1997).

10. R.P. Wei and D.G. Harlow, Model. Simul. Mater. Sci. 13, R33 (2005).

11. D.G. Harlow and R.P. Wei, Eng. Fract. Mech. 76, 695 (2009).
12. J.E. Bozek, J.D. Hochhalter, M.G. Veilleux, M. Liu, G. Heber, S.D. Sintay, A.D. Rollett, D.J. Littlewood, A.M. Maniatty, H. Weiland, R.J. Christ Jr., J. Payne, G. Welsh, D.G. Harlow, P.A. Wawrzynek, and A.R. Ingraffea, Model. Simul. Mater. Sci. 16, 065007 (2008).

13. J. Hochhalter, D. Littlewood, R. Christ Jr., M. Veilleux, J. Bozek, A. Ingraffea, and A. Maniatty, Model. Simul. Mater. Sci. Eng. 18, 045004 (2010).

14. J.D. Hochhalter, D.J. Littlewood, M.G. Veilleux, J.E. Bozek, A.M. Maniatty, A.D. Rollett, and A.R. Ingraffea, Model. Simul. Mater. Sci. Eng. 19, 035008 (2011)

15. S.R. Yeratapally, M.G. Glavicic, M. Hardy, and M.D. Sangid, Acta Mater. 107, 152 (2016).

16. W.D. Musinski and D.L. McDowell, Acta Mater. 112, 20 (2016).

17. G.M. Castelluccio and D.L. McDowell, Int. J. Fatigue 82, 521 (2016).

18. A. Rovinelli, Y. Guilhem, H. Proudhon, R.A. Lebensohn, W. Ludwig, and M.D. Sangid, Model. Simul. Mater. Sci. Eng. 25, 045010 (2017)

19. M.D. Sangid, Int. J. Fatigue 57, 58 (2013). Fatigue and Microstructure: A special issue on recent advances.

20. D. McDowell and F. Dunne, Int. J. Fatigue 32, 1521 (2010). Emerging Frontiers in Fatigue.

21. G.M. Castelluccio, W.D. Musinski, and D.L. McDowell, Curr. Opin. Solid State Mater. Sci. 18(4), 180 (2014). Slip Localization and Transfer in Deformation and Fatigue of Polycrystals.

22. H.-J. Christ, C.-P. Fritzen, and P. Köster, Curr. Opin. Solid State Mater. Sci. 18, 205 (2014). Slip Localization and Transfer in Deformation and Fatigue of Polycrystals.

23. A.D. Spear, J.D. Hochhalter, A.R. Cerrone, S.F. Li, J.F. Lind, R.M. Suter, and A.R. Ingraffea, Fatigue Fract. Eng. Mater. Struct. 39, 737 (2016).

24. T.J. Turner, P.A. Shade, J.V. Bernier, S.F. Li, J.C. Schuren, P. Kenesei, R.M. Suter, and J. Almer, Metall. Mater. Trans. A 48, 627 (2017)

25. A. Spear, S. Li, J. Lind, R. Suter, and A. Ingraffea, Acta Mater. 76, 413 (2014)

26. A. Spear, Numerical and Experimental Studies of ThreeDimensional Crack Evolution in Aluminum Alloys: Macroscale to Microscale. $\mathrm{PhD}$ thesis, Cornell University, 2014

27. K. Matouš and A. Maniatty, Int. J. Numer. Methods Eng. 60, $2313(2004)$

28. J.E. Warner, G.F. Bomarito, G. Heber, and J.D. Hochhalter, NASA / TM-2016-219180 (2016).

29. D. Raabe, M. Sachtleber, Z. Zhao, F. Roters, and S. Zaefferer, Acta Mater. 49, 3433 (2001).

30. R. Statistical Package, $R:$ A Language and Environment for Statistical Computing. Vienna, Austria: $R$ Foundation for Statistical Computing (2009).

31. P.I. Good, Permutation, Parametric and Bootstrap Tests of Hypotheses (Springer, Berlin, 2010). 